\title{
Lipid digestion and effects of diets rich in lipids on carbohydrate and nitrogen digestion. A review *
}

\author{
M Doreau, A Ferlay
}

INRA, Laboratoire Sous-Nutrition des Ruminants, Theix, 63122 Saint-Genès-Champanelle, France

This review deals with ruminal metabolism and intestinal digestion of lipids, and with the consequences of lipid supplementation on carbohydrate and nitrogen digestion. Ruminal hydrolysis of lipids and hydrogenation of polyunsaturated fatty acids are both extensive; generally $60-90 \%$ of linoleic acid and $80-100 \%$ of linolenic acid are hydrogenated. This process is independent of fatty acid intake, but is less complete for diets rich in concentrates. This effect could be due to modifications of the microbial ecosystem and/or the activity of enzymes at low pH. Hydrogenation of fatty acids can be reduced by the use of protein-coated lipids, but not by saponification. Duodenal flow of fatty acids is generally higher than fatty acid intake, due to lipid microbial synthesis, but may be lower, especially in diets rich in fat. The causes of this fatty acid disappearance are discussed. Absorption of long-chain fatty acids does not occur in the rumen. Mechanisms of intestinal absorption are the same as in monogastric animals, except that in ruminants, lipids reach the intestines in an unesterified form and are adsorbed on particles. Intestinal digestibitity of fatty acids varies between 65 and $90 \%$ in most experiments and is not related to fatty acid intake or composition, contrary to data based on digestibility measurements in the total tract. C16 and $\mathrm{C}_{18}$ fatty acids are more digestible than fatty acids with shorter or longer chains. Stearic acid is less digestible than $\mathrm{C} 18$ acids with 1 or 2 double bonds, but the difference is less marked than in monogastric animals. The synthesis of fatty acids occurs in the large intestine to a small extent.

The main effects of lipid addition are the ruminal decrease in fibre digestion and methanogenesis, the increase in propionic acid proportion in VFA and the drop in organic matter digested in the rumen. This is the result of important modifications of microbial ecosystem, especially a decrease of protozoa number and cellulolytic bacteria. Ruminal degradation of starch is not modified. Disturbances of ruminal carbohydrate digestion are the highest with polyunsaturated fatty acid supplementation, and generally with diets rich in starch. Ruminal nitrogen degradation, measured in situ and in vivo, and synthesis, measured in vivo, vary only to a small extent. They were not modified even if fatty acid intake was $10 \%$ of dry matter intake. Efficiency of $\mathrm{N}$ microbial synthesis increases if the organic matter digested in the rumen sharply decreases. Negative interactions between lipid and carbohydrate digestion are known to be reduced by a calcium supply. The positive effect of calcium was firstly attributed to the formation of calcium salts. Recent data show the low extent of salt formation and suggest an effect of calcium in its ionic form. Ionized calcium (but not ionized magnesium) may be a limiting factor for the action of microbes in organic matter degradation, probably by adhesion of bacteria to particulate matter.

\footnotetext{
* Part of this review has been published previously in Anim Feed Sci Technol (1994) 45, 379-396.
} 